\title{
Limits for the application of spectroscopic mode ID methods
}

\author{
W. Zima ${ }^{1}$, K. Kolenberg ${ }^{1,2}$, M. Briquet ${ }^{2}$ and M. Breger ${ }^{1}$ \\ ${ }^{1}$ Institut für Astronomie, Universität Wien, Türkenschanzstr. 17, 1180 Wien, Austria \\ e-mail: zima@astro.univie.ac.at \\ ${ }^{2}$ Instituut voor Sterrenkunde, Celestijnenlaan 200B, 3001 Heverlee, Belgium
}

\begin{abstract}
Spectroscopic mode identification techiques, which monitor intensity variations across an absorption line, provide the possibility of determining the quantum numbers 1 and $\mathrm{m}$, the inclination and the intrinsic pulsation amplitude of a star. Of course, the uncertainties of the mode identification are dependent on the quality of the observations and the identification method applied. We have focused on the Pixel-by-pixel method/Direct line profile fitting (Mantegazza 2000) and the Moment method (Balona 1987, Briquet \& Aerts 2003) for pinpointing mode parameters and tested the impact of various observational effects and stellar properties on the identification.
\end{abstract}

Keywords. Line: profiles, methods: numerical, techniques: spectroscopic, stars: individual: (FG Vir, 44 Tau), stars: oscillations

\section{Pulsational model of the tests}

The line profiles we used for this analysis were computed by integrating over the pulsational velocity field on the stellar surface, consisting of a 64800-element grid and taking a Gaussian as the intrinsic line profile. The resulting velocities were calculated by summing the contribution of every single pulsation mode. The model is based on the descriptions given by Schrijvers et al. (1997) and Balona (2000) and takes into account the effect of the Coriolis force, first and second order limb darkening, and temperature variations.

\section{Radial velocity limit for Doppler imaging}

A major limit for the application of both the Intensity Period Search Method (Schrijvers \& Telting 1999) and the Pixel-by-pixel method is set by the pulsational amplitude and the resulting projected radial velocity (RV) of the pulsation modes. If the RV of a certain mode becomes a significant fraction of $v \sin i$, the Doppler Imaging of the stellar surface is nonlinear. In a multiperiodic star this prevents a correct identification of its pulsation modes due to phase- and amplitude-distortion if one or more modes have RV amplitudes above $0.2 v \sin i$.

\section{Phase smearing}

An integration time being a significant fraction of the measured sinusoidal signals period leads to phase smearing, inducing a damping of the observed amplitude. Therefore, it is important to keep the integration time as short as possible. We examined the influence of phase smearing on the line profile variations for different values of the relative integration time to the pulsation period. For modes having a pulsation period of five 


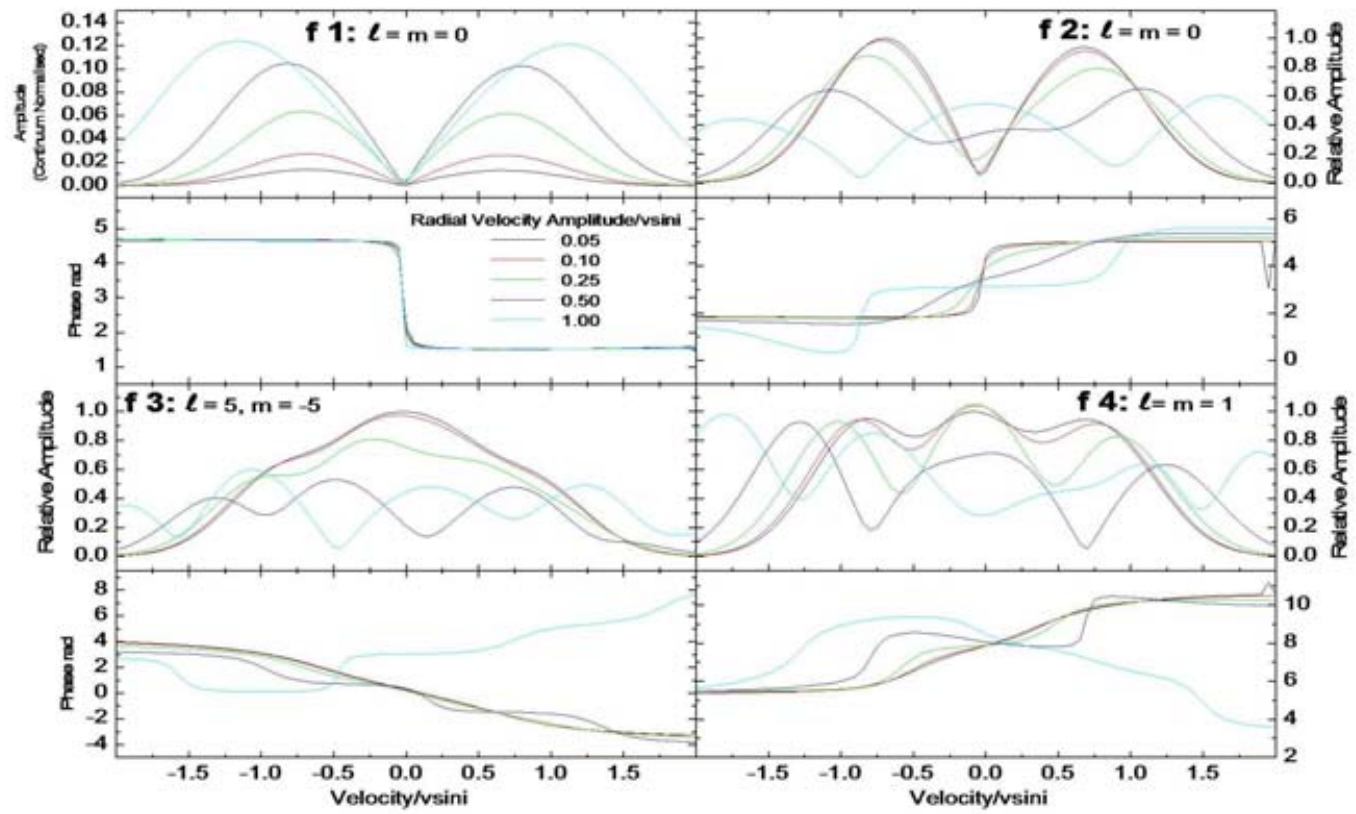

Figure 1. Influence of a mode with a large radial velocity on the amplitude and phase across the line of other simultaneously excited pulsation modes. When applying Fourier techniques and Least-Squares fitting, respectively, on the Pixel-by-pixel variations of a line to decompose the pulsational content of every single pulsation mode, nonlinearities caused by modes having radial velocity amplitudes (RVA) a significant fraction of $v \sin i$ can prevent mode identification. In this test we simulated line profile variations of four simultaneously excited pulsation modes. We subsequently increased the amplitude of only $f_{1}$ to study the impact on the three other modes, which have radial velocity amplitudes below $0.01 v \sin i$. For a ratio of the RVA to the projected rotational velocity of 0.2 , the amplitude and phase distributions for all three modes are significantly distorted and a correct identification is hindered. For $f_{3}$ even the sign of $\mathrm{m}$ cannot be stated, if RVA $/ v \sin i$ reaches one.

times the integration time, a significant (0.93) reduction of the observed amplitudes occurs, which mainly affects the derived intrinsic pulsational amplitude and only has minor influence on the identification of 1 and the star's aspect angle.

\section{A Hare-and-hound test}

We have carried out a Hare-and-hound test on synthetic spectroscopic data to examine the capability and reliability of the Pixel-by-pixel method as well as the Moment Method on low order pulsation modes, such as detected in FG Vir or 44 Tau.

The first mode identification technique we tested makes use of the Pixel-by-pixel method, where briefly summarized, the pulsational content of every single oscillation mode is detected by Fourier-analysing the variations of each wavelength bin, extracted by Least-Squares Techniques (decomposition), treated as mono mode pulsation and fitted with theoretical profiles by means of a genetic algorithm.

The Moment Method makes use of that line profile variations can be sufficiently described by three moments, which are integrated over the line and fitted with theoretically determined moments.

We also tested a combined method by fitting the moments of the decomposed line 

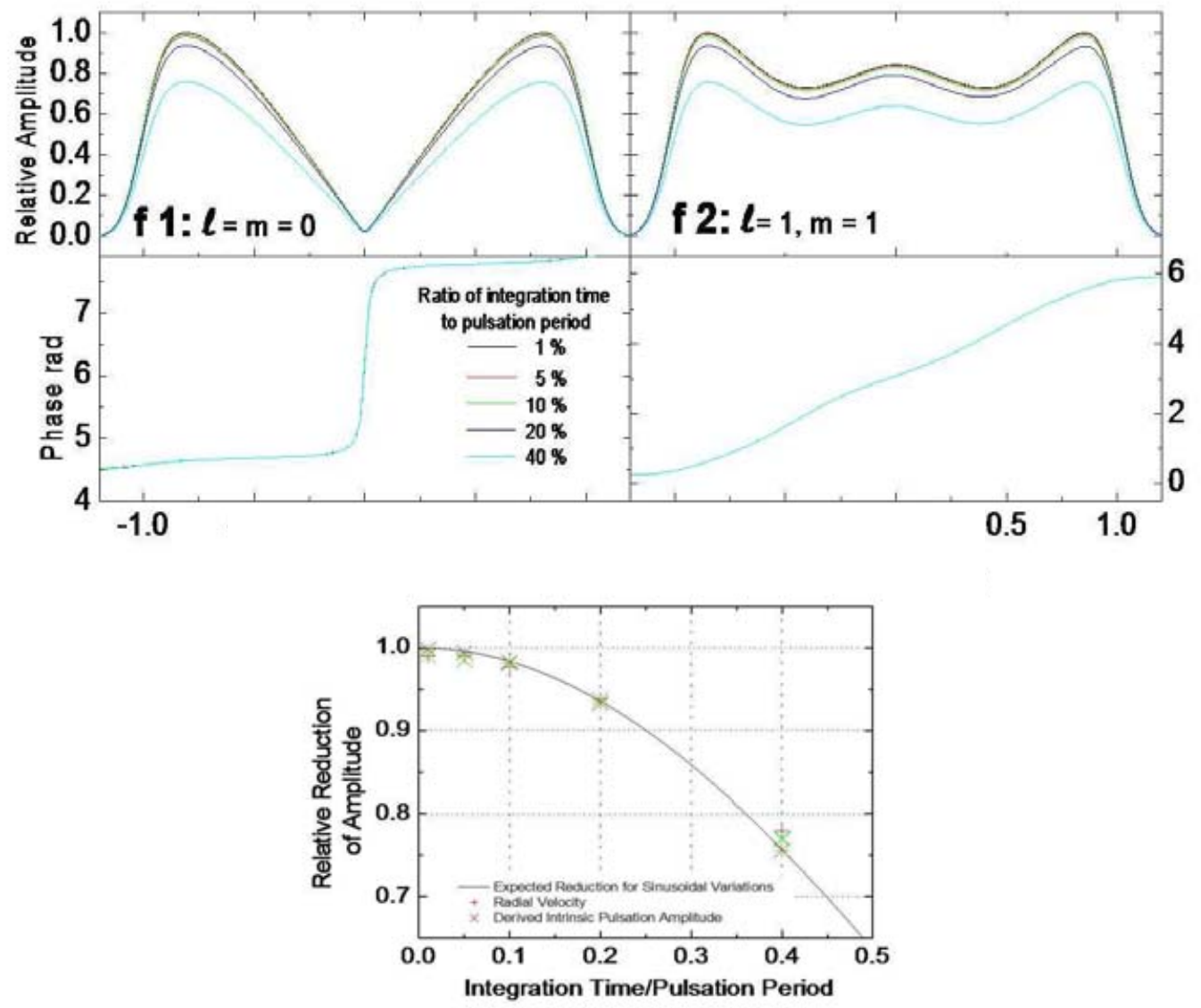

Figure 2. Effect of phase smearing on amplitude and phase across the line for different pulsation modes. The left panels show the relative amplitude and phase across a line for different integration times. The phase smearing primarily affects the amplitude of the pixel variations, which in turn gives rise to an underestimate of the intrinsic pulsation amplitude, a wrong determination of the harmonic degree $l$ or the stellar inclination. Due to that the phase distribution is independent of the smearing, no additional error on the azimuthal degree $m$ is induced. The bottom panel shows the relative amplitude reduction of the First Moment (radial velocity) and the intrinsic pulsation amplitude $(\mathrm{DR} / \mathrm{R})$, derived from a direct line profile fit $\left(f_{1}\right.$ in red, $f_{2}$ in green). Both values fit to the expected trend. The behaviour of the two illustrated modes is representative for other values of $l$ and $m$.

profiles. This technique enabled the fitting of all detected modes simultaneously in much shorter computational time.

We created realistic data of a FG Vir-like star having a $v \sin i$ of about $20 \mathrm{~km} \mathrm{~s}^{-1}$, which exhibited pulsation mode parameters initially unknown to us. The simulation included the effect of phase smearing, the noise of real observational data and observation times of the 2002 FG Vir campaign of the Delta Scuti Network (Zima et al. 2003).

All applied identification methods yielded consistent results. Whereas the azimuthal order $\mathrm{m}$ could be determined unambigously, it was not possible to discriminate between different $l$ values.

The inclination was determined with an error of $4^{\circ}$. Fitting all modes simultaneously gives much more consistent results, due to that for continuous parameters, such as the 


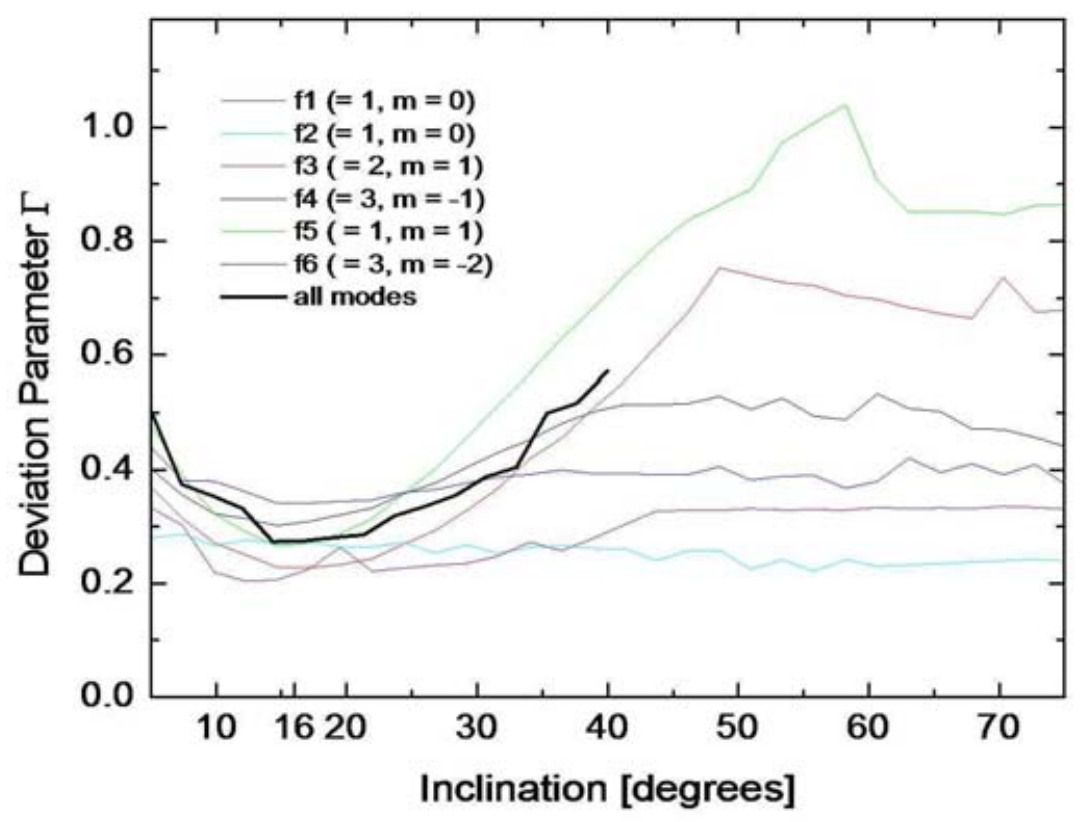

Figure 3. Dependence of the deviation parameter (arbitrary units) derived from the line profile fits of the inclination of the model. For every inclination only the lowest deviation parameter, representing the difference between the observed and modelled line profiles, for the specific mode is shown. An important feature is that for the two axisymmetric modes we derive significantly wrong inclinations, which mainly is a consequence of a low dependence of $\Gamma$ on the aspect angle. In contrast, the non-axisymmetric modes all show a minimum of the deviation parameter at about $16^{\circ}$, the correct value of the input model. An improvement is made by fitting all six modes simultaneously with common inclination.

inclination, only one value is derived. Unfortunately, such a multimode fit is computationally much more time-demanding and can only applied on a limited number of modes.

A more detailed description of this test can be found in Zima et al. (2004).

\section{Mode identification of FG Vir}

We observed line profile variations of the $\delta$ Scuti star FG Vir (HD 106384, $V=6.6$, $\mathrm{A} 5 \mathrm{~V}$ ) in the framework of a global spectroscopic/photometric observing campaign (ESO, Chile, McDonald Obs., USA, Tautenburg, Germany, SAAO, South Africa, Mt. John Obs., New Zealand) which lasted from 2002 February to April, yielding 122 hours of high resolution spectra. The mode identification of these spectra was successfully acquired by the above described methods.

We were able to detect 13 independent pulsation frequencies between 9.1 and $24.2 \mathrm{~d}^{-1}$ in the spectra, which all have a counterpart among the 60 frequencies detected photometrically during this campaign (Breger et al. 2004).

The results of the line profile analysis showed that eight modes have axisymmetric character $(\mathrm{m}=0)$ and 5 modes have an azimuthal order between -2 and 1 . All detected modes are low order modes with $l \leqslant 3$, which mainly is caused by this stars low projected rotational velocity of about $21 \mathrm{~km} \mathrm{~s}^{-1}$. We were also able to derive the inclination of FG Vir $\left(i=20 \pm 5^{\circ}\right)$ and through comparison of photometric light variations in Strömgren filters with the intrinsic pulsation amplitude we derived the ratio of flux perturbation to 


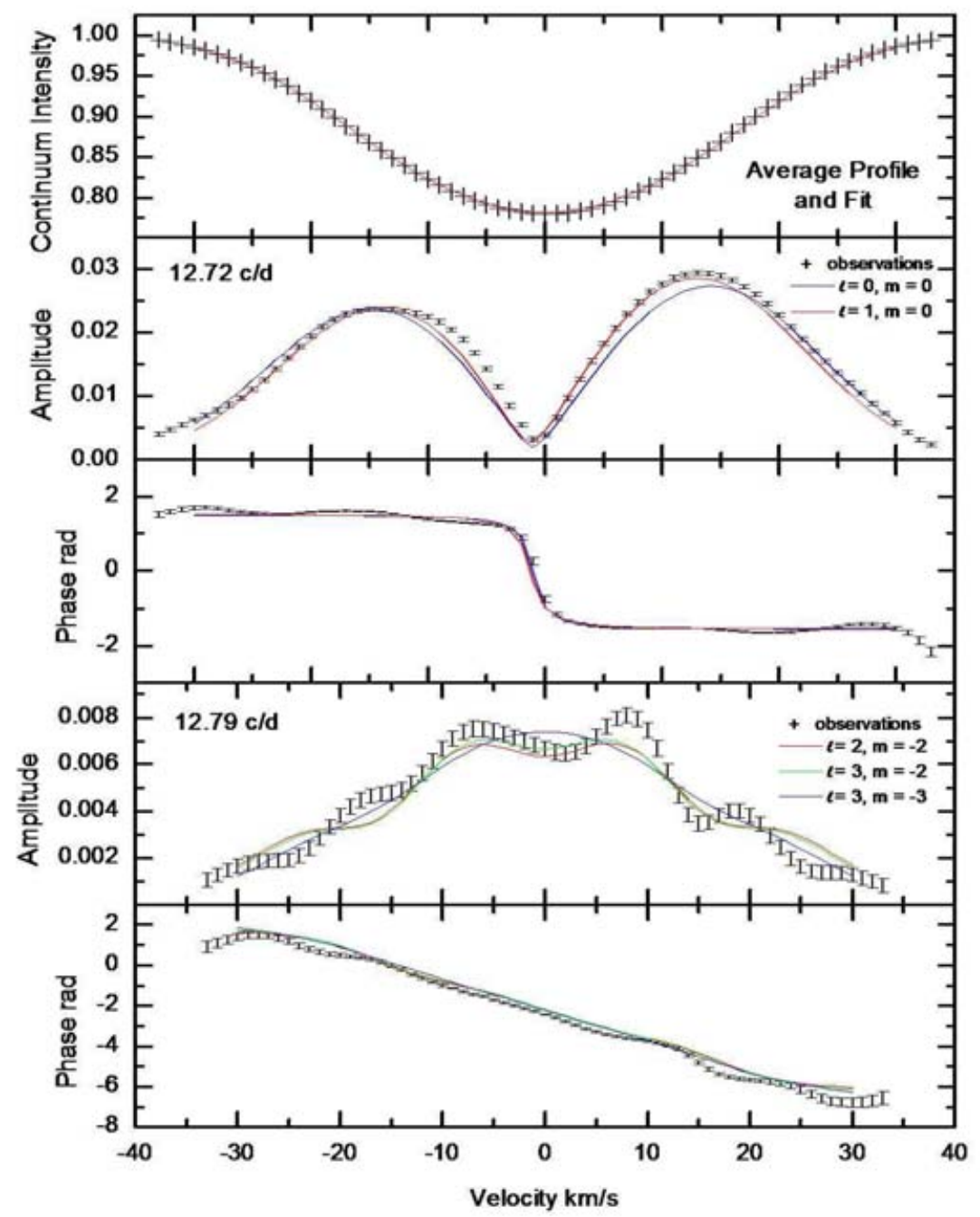

Figure 4. Observed and modelled amplitude and phase across the line (combination of $3 \mathrm{Fe} \mathrm{I}$ lines) for two pulsation modes of FG Vir.

radial displacement, which is very sensitive to convection, and thus enables the selection between different convective models (Daszynska-Daszkiewicz et al. 2003).

\section{Conclusions}

- mode ID Methods relying on Doppler Imaging fail for a multiperiodic star, having a mode with a ratio of the radial velocity amplitude to $v \sin i>0.2$

- long integration times reduce the observed amplitudes by smearing must be taken into account when deriving the intrinsic pulsational amplitudes

- the Pixel-by-pixel and Moment methods give consistent results for low order pulsation modes in slowly rotating stars but: problems with the identification of the harmonic degree $l$

- successful spectroscopic mode ID of FG Vir, new theoretical models will be proposed soon. 


\section{Acknowledgements}

This work is supported by the Austrian Fonds zur Frderung der wissenschaftlichen Forschung (FWF) within the project Stellar Seismology (P14546-Phy). M. Briquet is Postdoctoral Fellow of the Fund for Scientific Research, Flanders.

\section{References}

Balona, L.A. 1987, MNRAS 224, 41

Balona, L.A. 2000, ASP Conf. Ser. 210, 170

Breger, M., Rodler, F., Pretorius M.L., Martín-Ruiz, S., Amado, P.J. et al. 2004, AESA 419, 695

Briquet \& M., Aerts, C. 2003, A\&BA 398, 687

Daszynska-Daszkiewicz, J., Dziembowski, W.A., \& Pamyatnykh, A.A. 2003, A\&EA 407, 999

Schrijvers C., Telting, J.H., Aerts, C., Ruymaekers, E., \& Henrichs, H.F. 1997, A\&SAS 121, 343

Schrijvers, C. \& Telting, J.H. 1999, A\&3A 342, 453 